\title{
Identification of a new cystic fibrosis transmembrane regulator mutation in a severely affected patient
}

\author{
E. Spitzer*, D. Staab* , R. Hanke*, U. Wahn\#, R. Grosse*
}

\begin{abstract}
Identification of a new cystic fibrosis transmembrane regulator mutation in a severely affected patient. E. Spitzer, D. Staab, R. Hanke, U. Wahn, R. Grosse. (C) ERS Journals Ltd 2002.

ABSTRACT: By using a combination of multiplex polymerase chain reaction and allele-specific labelled probes, the oligo-ligation assay is designed to detect known cystic fibrosis transmembrane regulator mutations. This study shows that this assay may also be useful to detect new mutations.

The second child of a family of Bosnic origin showed all the symptoms of intestinal and pulmonary manifestations of cystic fibrosis. No signal could be obtained for the allele-specific probe $1898+1 G>A$. This could be explained by a nearby localized sequence change that prevented polymerase chain reaction primers or oligonucleotide probes from binding to the target sequence. Indeed, sequence analysis revealed a new 1894G $>$ T exchange (Glu587Stop). Both parents and the healthy brother carried this mutation. Thus, the index patient was homozygous for $1894 G>T$, which was inherited from both parents.
\end{abstract}

Eur Respir J 2002; 19: 374-376.
*Institute of Medical Molecular Diagnostics Ltd, Berlin, and \#Christiane Herzog Centre, Chest Clinic Heckeshorn, Berlin, Germany.

Correspondence: E. Spitzer, Institute of Medical Molecular Diagnostics, Schoenstrasse 90, 13086 Berlin, Germany.

Fax: 49 309209071

E-mail: labor@immdonline.com

Keywords: Cystic fibrosis, cystic fibrosis transmembrane regulator gene mutation, oligo-ligation assay

Received: July 22001

Accepted after revision July 312001
Cystic fibrosis (CF) is an inherited disorder that is characterized by respiratory disease and pancreatic dysfunction [1]. CF results from mutations in the gene encoding the $\mathrm{CF}$ transmembrane conductance regulator (CFTR). The CF phenotype can be caused by $>900$ different mutations in the CFTR gene [2]. With the exception of the more common 3-base pair deletion in exon $10(\Delta \mathrm{F} 508)$, the vast majority of mutations are rare or restricted to certain populations. In order to make an efficient screening for the more common known mutations possible, the oligo-ligation assay (OLA) has recently been introduced [3, 4]. By using a combination of multiplex polymerase chain reaction (PCR) and allele-specific fluorescentlylabelled probes ligated to CFTR mutation sites, deoxyribonucleic acid (DNA) fragments harbouring each of the 31 more common mutations can be produced. Identification of a CFTR genotype is achieved by size separation and gene scanning on an ABI 377 device (Applied Biosystems, Foster City, CA, USA). The OLA assay is designed to detect known mutations for which specific probes must be available. Nevertheless, this paper shows that this assay has made the detection of a new nonsense mutation possible in a family of Bosnic origin.

\section{Case report}

A family of Bosnic origin with two children was studied. After normal delivery, the second child showed symptoms of dyspnoea, dilated bowel and intestinal obstruction at the age of $3 \mathrm{~h}$. After meconium aspiration, connatal pneumonia was diagnosed. The child was treated with antibiotics and the intestinal obstruction was resolved with enemas. At the age of 4 months, he was re-admitted to the hospital because of an atelectatic pneumonia. At that time, the diagnosis of $\mathrm{CF}$ was verified by a pathological sweat test and he was referred to a $\mathrm{CF}$ centre. The second and third sweat test were found to be pathological (sodium: 74 and $83 \mathrm{mmol} \cdot \mathrm{L}^{-1}$ ) and pancreatic insufficiency was diagnosed. The child showed all symptoms of intestinal and pulmonary manifestation of CF. The elder brother was healthy and there was no history of CF or early deaths in childhood in the family, but the grandparents of both families were born in the same village.

DNA samples were obtained from all members of the family from dried blood stains prepared as previously described [5]. They were routinely examined by means of the CF assay kit from Applied Biosystems.

\section{Discussion}

Studies carried out in southern European populations, including the former Yugoslavia, identified $\Delta \mathrm{F} 508, \mathrm{G} 542 \mathrm{X}, \mathrm{G} 551 \mathrm{D}, 621+1 \mathrm{G}>\mathrm{T}, \mathrm{W} 1282 \mathrm{X}$ and $\mathrm{N} 1303 \mathrm{~K}$ as the most common CF mutations [6, 7]. To the authors' knowledge, no data has been published about the Bosnic population.

In order to screen for CFTR mutations, the DNA 

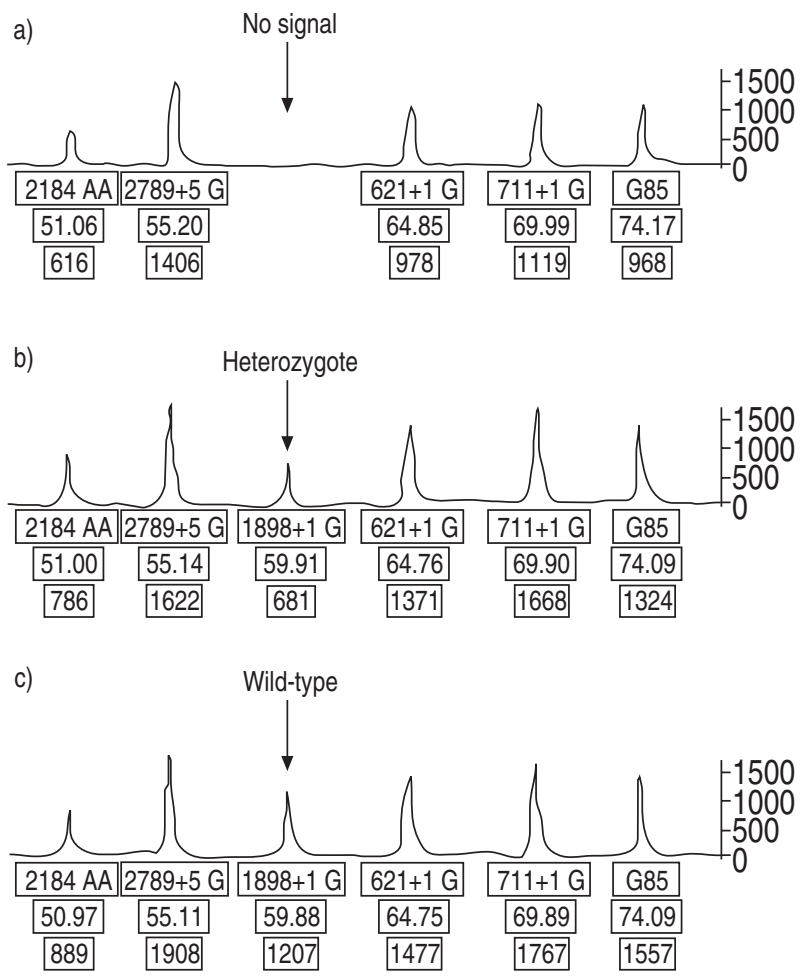

Fig. 1.-Oligo-ligation-assay. The plots of the electropherograms for six different mutation sites in the cystic fibrosis transmembrane regulator (CFTR) gene are shown. The numbers in the boxes indicate the mutation site, the size of the fragment and the height of the peak. a) The plot obtained from the index patient's sample. No signal could be detected for $1898+1 \mathrm{G}$ in intron 12 of the CFTR gene (arrow). b) The plot obtained from the blood sample of the index patient's father. The signal obtained for $1898+1 \mathrm{G}$ was reduced in comparison with the five other mutation sites of the same panel and the wild-type signal for $1898+1 \mathrm{G}$ shown in c). The same results were obtained with blood samples from the mother and the healthy brother of the patient (data not shown). c) The plot obtained using a wild-type control sample.

sample obtained from the index patient was analysed by means of the OLA assay. For 30 of the allelespecific probes the assay revealed no differences to the wild-type sequence (fig. 1). However, no signal could be obtained for either the wild-type or the mutated site of the allele-specific probe $1898+1 \mathrm{G}>\mathrm{A}$ in intron 12 [8]. This may be explained by a sequence change that prevented PCR primers from binding to the target sequence, thus inhibiting amplification of the DNA fragment harbouring the $1898+1 \mathrm{G}>\mathrm{A}$ genotype. Alternatively, a sequence alteration in the binding domain for the oligonucleotide ligation probes could have prevented fragment analysis. Nevertheless, the data suggest a sequence deviation in the vicinity of the site $1898+1 \mathrm{G}$. To test this assumption, exon 12 , including the exon-intron boundaries, was sequenced. Sequence analysis showed a $\mathrm{G}>\mathrm{T}$ exchange at the base position 1894, which generated a termination signal at amino acid position 587 (Glu>Stop; fig. 2).

The lack of an OLA signal at position $1898 \mathrm{G}>\mathrm{A}$ in exon 12 could therefore be interpreted as a heterozygous $\mathrm{G}>\mathrm{T}$ exchange associated with a simultaneous deletion in the region of exon/intron 12 on the other allele, or, alternatively, might have been due to a
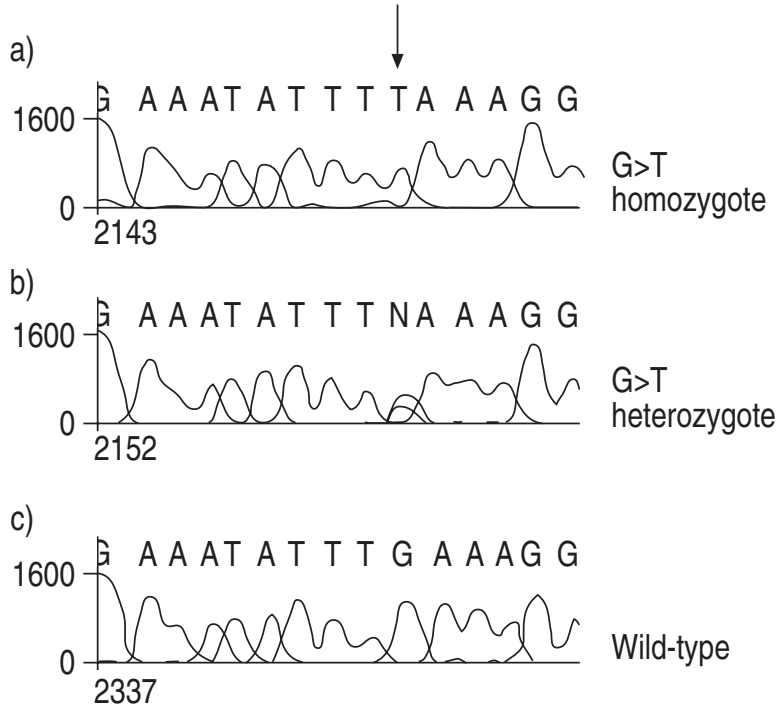

Fig. 2.-Sequencing analysis. The results of sequencing polymerase chain reaction fragments including exon and intron 12 of the cystic fibrosis transmembrane regulator gene. a) The sequence from the index patient's sample shows a homozygous G>T exchange at position 1894. b) The sequence from the sample of the index patient's father. The heterozygous exchange $\mathrm{G}>\mathrm{T}$ at nucleotide position 1894 was found and confirmed for the patient's mother and the healthy brother (data not shown). c) The sequence from a wild-type control sample.

homozygous $\mathrm{G}>\mathrm{T}$ exchange at position 1894. Both parents and the healthy brother of the index patient were therefore examined for the presence of $1894 \mathrm{G}>$ T. All three individuals were found to be heterozygous carriers of the mutation $1894 \mathrm{G}>\mathrm{T}$ (figs. 1 and 2). Thus, the index patient inherited the mutation from both parents and was classified as homozygous for the mutation $1894 \mathrm{G}>\mathrm{T}$ (Glu587Stop).

The data presented show the sensitivity and efficiency of the oligo-ligation assay for detecting single-base substitutions. If under routine conditions the allelic ligation probes fail to produce specific fragments and fluorescence signal using the oligoligation assay, it is therefore straightforward to test for sequence variants by sequencing the respective deoxyribonucleic acid fragment or exon. Finally, it would be interesting to further explore the frequency of the mutation $1894 \mathrm{G}>\mathrm{T}$ among the Bosnic population. This can be efficiently achieved through the use of the oligo-ligation assay.

\section{References}

1. Schwiebert EM, Benos DJ, Fuller CM. Cystic fibrosis: a multiple exocrinopathy caused by dysfunctions in a multifunctional transport protein. Am J Med 1997; 104: 576-590.

2. www.genet.sickkids.on.ca/cftr-cgi-bin/FullTable. Date accessed: September 92000.

3. Grossmann PD, Bloch W, Brinson E, et al. High density multiplex detection of nucleic acid sequences: oligonucleotide ligation assay and sequence-coded separation. Nucleic Acids Res 1994; 22: 4527-4534. 
4. Applied Biosystems. Cystic Fibrosis Assay: Manual 1997.

5. Spitzer E, Abbaszadegan MR, Schmidt F, et al. Detection of BRCA1 and BRCA2 mutations in breast cancer families by a comprehensive two stage screening procedure. Int $J$ Cancer 2000; 85: 474-481.

6. Kanavakis E, Tzetis M, Antoniadi T, et al. Mutation analysis of ten exons of the CFTR gene in Greek cystic fibrosis patients. Hum Genet 1995; 96: 364-366.
7. Estivill X, Bancells C, Ramos C. Geographic distribution and regional origin of 272 cystic fibrosis mutations in European populations. The Biomed CF Mutation Analysis Consortium. Hum Mutation 1997; 10: $135-154$.

8. Strong TV, Smit LS, Nasr S, et al. Characterization of an intron 12 splice donor mutation in the cystic fibrosis transmembrane conductance regulator (CFTR) gene. Hum Mutation 1992; 1: 380-387. 